

\section{polisemie}

\section{I}

\section{1}

Direzione

Stefano Milonia (University of Warwick)

Comitato scientifico

Giulia Bassi (Università degli Studi di Siena)

Mario Cianfoni (Sapienza Università di Roma)

Stefano Colangelo (Alma Mater Studiorum - Università degli Studi di Bologna)

Luigi Marinelli (Sapienza Università di Roma)

Carlo Pulsoni (Università degli Studi di Perugia)

Niccolò Scaffai (Università degli Studi di Siena)

Costantino Turchi (Sapienza Università di Roma)

Fabio Zinelli (École Pratique des Hautes Études)

\section{Redazione}

Stefano Bottero (Università Ca' Foscari Venezia)

Mattia Caponi (Sapienza Università di Roma)

Carlo Londero (Università degli Studi di Udine)

Giorgio Tranchida (Alma Mater Studiorum - Università degli Studi di Bologna)

Samuele Maria Visalli (Sapienza Università di Roma)

Arianna Saggio (Sapienza Università di Roma)

Andrea Bongiorno (Aix-Marseille Université)

Giulia Boitani (University of Cambridge)

Alessandra Frustaci

Polisemie è una rivista annuale pubblicata dalla University of Warwick Press.

Gli articoli pubblicati nella sezione Saggi sono sottoposti a double-blind peer review.

Licenza Creative Commons - Attribuzione (CC-BY 4.0).

ISSN: 2634-1867

DOI: 10.31273 /polisemie.v1

Immagine copertina: Serse Luigetti 


\title{
INTUITISME ET ART BRUT DANS LA POÉSIE D'HÉDI BOURAOUI
}

\author{
Simone Grossman
}

Intuitire... c'est ne point languir

Après le sens

[...]

Intuitire... c'est souvent sentir

Les choses... les événements

Arriver de surprises...

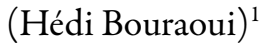

Isabelle Danto, critique d'art, attribue la fascination actuelle de l'art brut à la saisie immédiate du geste créateur et à une conception élargie de l'œuvre d'art. ${ }^{2} \mathrm{La}$ présente étude se préoccupe de la fonction de l'art brut dans la poésie d'Hédi Bouraoui, poète canadien d'origine tunisienne adhérant à l'intuitisme, mouvement artistique et littéraire des années 2000 fondé par Éric Sivry et Sylvie Biriouk. L'intuitisme, basé sur la «corrélation » entre le moi intime et le « hors-moi », participe de la tendance contemporaine à de « nouveaux élans vers l'altérité ». ${ }^{3}$ Les intuitistes aspirent à créer un «espace pluriartistique où les arts se fiancent pour dépasser les anciennes frontières $\gg .{ }^{4} \mathrm{La}$ convergence de l'intuitisme et de l'art brut dans la poésie de Bouraoui sera au centre de notre propos. Comme le montrera l'examen de poèmes illustrés par Claudine Goux, Dodik Jégou, Adam Nidzgorski et Rêva Rémy, ${ }^{5}$ l'intuition coïncide chez Bouraoui avec la «pure et authentique impulsion créative $\gg$ d'où s'origine l'art selon Jean Dubuffet. ${ }^{6}$

\footnotetext{
${ }^{1}$ Hédi Bouraoui, En amont de l'intuition. Poèmes, Toronto, CMC Éditions, 2013, p. 12.

${ }^{2}$ Isabelle Danto, Quand l'art brut redessine l'art contemporain, in « Esprit », (Mars-Avril 2015).

${ }^{3}$ Éric Sivry, Pour un art de l'intuition. Manifeste de l'intuitisme, Paris, Hermann Éditeurs, 2016, pp. 34-35.

${ }^{4}$ Ibid., p. 94.

${ }^{5}$ Claudine Goux, Dodik Jégou et Adam Nidzgorski sont des artistes de la Création Franche. L'expression a été créée en 1989 par Gérard Sendrey, suite à l'interdiction du musée de Lausanne, dépositaire de la collection de Jean Dubuffet, d'utiliser l'expression art brut. Le Musée de la Création Franche - Art brut et apparentés se trouve à Bègles près de Bordeaux. « Hors normes » est une autre appellation de l'art brut; Raw Art et Outsider Art sont les synonymes anglais d'art brut. Rêva Rémy, artiste indépendante, peint dans la spontanéité.

${ }^{6}$ Jean Dubuffet, L'homme du commun à l'ouvrage, Paris, Gallimard, 1991 [1973], p. 67.
} 
Notre réflexion s'étayera sur la théorie de l'intermédialité relative à l'inclusion de tableaux dans les textes littéraires. ${ }^{7}$ Selon Liliane Louvel, la coprésence de l'écrit et de la peinture a pour effet de changer le statut du texte. ${ }^{8}$ Nous verrons que la contiguïté entre le visuel et le verbal s'origine de l'intuition et que, corroborant l'insertion des tableaux, les références textuelles à l'art brut et l'emprunt de l'écrit aux techniques picturales font dialoguer les expressions artistiques. Bouraoui précise que les dessins et les tableaux accompagnant ses poèmes ne sont pas des illustrations. ${ }^{9}$ L' « unité indissoluble de texte(s) et image(s) dans laquelle ni le texte ni l'image n'ont de fonction illustrative $\gg,{ }^{10}$ caractérisant l'iconotexte, s'applique à l'ensemble des poèmes de Bouraoui en interaction avec les œuvres picturales. En nous fondant sur le principe de Louvel pour qui « à l'origine du tableau se trouve une pensée $[. .$.$] mise en acte » et « retraduite [. .$.$] par une autre pensée { }^{11}$ nous constaterons que la rencontre entre les expressions artistiques et les cultures, est motivée chez Bouraoui par la représentation de l'intuition en acte. ${ }^{12}$

Le premier volet de notre étude sera consacré à la similarité entre le narratoème bouraouien et l'art brut auquel il se réfere. Les développements suivants seront consacrés à la collaboration du poète avec Claudine Goux. Puis nous examinerons l'iconotexte sur la première de couverture de Traversées, ${ }^{13}$ composé du titre surimposé au tableau de Rêva Rémy, Le Temple Voilier. Au stade suivant, le dessin d'Adam Nidzgorski, sur la page liminaire de Transit en Art Majeur, mènera à définir la nomadanse. En conclusion, le processus créateur qui se dégage de la coprésence des poèmes et de l'art brut sera brièvement résumé.

\section{Le narratoème bouraouien et les références à l'art brut}

Dans L'intuition à l'ouvre, préface à En amont de l'intuition, Bouraoui définit l'intuition comme la « source d'énergie émotionnelle [...] toujours présente dans

\footnotetext{
${ }^{7}$ Voir à ce sujet Peter Wagner, Icons - Texts - Iconotexts. Essays on Ekphrasis and Intermediality, Berlin-New York, Walter de Gruyter, 1996.

${ }^{8}$ Liliane Louvel, A reading event The Pictorial Third, in Sillages critiques, 21 (2016).

${ }^{9}$ Hédi Bouraoui, Inside Faces-Visages du dedans. Visiotexts/Visionèmes Paintings by/Peintures de Micheline Montgomery, Toronto, CMC Éditions, 2014, p. 14.

${ }^{10}$ Michael Nerlich, Qu'est-ce qu'un iconotexte? Réflexions sur le rapport texte-image photographique dans La Femme se découvre d'Evelyne Sinnassamy, dans Iconotextes, sous la direction de Alain Montandon, Paris, Ophrys, 1990, pp. 255-302.

${ }^{11}$ Liliane Louvel, Le tiers pictural, Rennes, Presses Universitaires de Rennes, 2010, p. 53.

12 Comme en fait état Marilyne Bertoncini : «Sivry distingue l'intuitisme de la démarche philosophique intuitionniste de Kant ou Bergson, qu'il cite comme étant proches, tout en revendiquant la singularité de la démarche intuitiste » (Marilyne Bertoncini, Poésie-première 72 : l'intuitisme [Compte rendu], in Recours au Poème).

${ }^{13}$ Hédi Bouraoui, Traversées. Poèmes, Toronto, CMC Éditions, 2010.
} 
toute écriture créatrice ou toute peinture en élaboration », dont « aucun créateur ne peut se passer $\gg .{ }^{14}$ L'artiste ne retranscrit pas l'intuition. Au contraire, «l'intuition se manifeste dans son œuvre en créant selon les arts des traces particulières $\gg .{ }^{15}$ Dans la poésie de Bouraoui, les « traces » ou « résonances » de l'intuition, selon l'expression de Mario Selvaggio, ${ }^{16}$ en appellent à la peinture, à la danse et à la musique non moins qu'à l'écriture. «Les poètes intuitistes ne favorisent pas une langue, ils aiment les mêler toutes », dit Sivry selon qui Bouraoui « se situe parfaitement dans le mouvement de ce point de vue $\gg .{ }^{17} \mathrm{~L}^{2}$ « écriture sans frontières $\gg^{18}$ de Bouraoui, appartenant à trois cultures différentes, africaine, européenne et nord-américaine, mêle les langues et les genres. Sivry note que ses écrits se situent «dans la catégorie des ouvres intuitistes de l'interstitiel des rencontres $\gg .{ }^{19}$ L'intuition, invite au dialogue et à l'échange, s'avère être une disposition, voire «un état de création [...] régissant l'œuvre », précédant immédiatement l'acte créateur. ${ }^{20}$ Tel l'art brut, l'art intuitiste naît du silence de la pensée rationnelle pour l'artiste, poète ou peintre qui, en « état d'abandon total [...] laisse faire $» .{ }^{21}$ Bouraoui décrit l'avènement du poème qui prend forme « dans son chantournement interne » et rayonne « en objet d'art parfait », ${ }^{22}$ élimine le savoir établi au profit de la connaissance intuitive. L'influx de l'intuition, « sixième sens $\gg,{ }^{23}$ est saisi dans l'expression intuitiste, qu' elle soit verbale ou visuelle. Dans son étude sur En amont de l'intuition, Elizabeth Sabiston expose que l'intuition, ordonnatrice de l'impulsion, transbordant la création en nomadisme inter artistique, est la source du Transréel bouraouïen. Elle montre que l'écriture poétique, palliant à l'incapacité langagière, recourt à des néologismes pour traduire les sensations : les néologismes Intuitire, Intuité, Intuitionner modulent les effets de l'intuition, appartenant au même registre que Barbarituif ${ }^{24}$ faisant allusion à la poussée naturelle de la figue de Barbarie.

Bouraoui explicite dans l'avant-propos de NomadiVivance I la notion de narratoème, « mot-concept » issu de l'intuition du poète rapportant uniquement

\footnotetext{
${ }^{14}$ Hédi Bouraoui, L'intuition à l'euvre, in Id., En amont de l'intuition, pp. 6-8, citation à p. 6.

${ }^{15}$ Sivry, Pour un art de l'intuition, p. 25.

${ }^{16}$ Mario Selvaggio, Hédi Bouraoui, Passerelles. Poésie, Toronto, CMC Éditions, 2018 [Compte rendu], in « Revue européenne de recherches sur la poésie », 4 (2018), pp. 260-262.

${ }^{17}$ Sivry, Pour un art de l'intuition, p. 40-41.

${ }^{18}$ Ibid., p. 41.

${ }^{19}$ Ibid., p. 187.

${ }^{20}$ Ibid., pp. 80-81.

${ }^{21}$ Ibid., p. 90.

${ }^{22}$ Bouraoui, En amont de l'intuition, p. 6.

${ }^{23} \mathrm{Ibid}$.

${ }^{24}$ Voir l'article d'Elizabeth Sabiston, Upstream: Hédi Bouraoui's En amont de l'intuition, in « Revue CMC Review », 1 (2014), 1, pp. 1-9.
} 
les faits, à la manière d'un chroniqueur qui enregistre la réalité « en la scrutant bien en face, à l'état brut $\gg .{ }^{25} \mathrm{La}$ première moitié du néologisme évoque l'instabilité intrinsèque du réel. « Narrer les flux et reflux de la Vie |C'est les composer en mosaïques de l'Ecrit $\gg{ }^{26}$ Le poème transpose au plan verbal le chaos éclaté et mouvant de l'existence, reflétant le nomadisme physique et spirituel de l'artiste, ou nomaditude, ${ }^{27}$ dans un écrit rythmé par la danse au son de la musique. Il exprime la réalité de façon authentique, sans l'enjoliver: «Peu d'embellissement de l'écrit...livré dans ces textes dans son effet Brut $\gg \cdot{ }^{28}$ L'intégration à l'écrit de termes désignant les groupes et les tendances issus de l'art brut, tels « Création franche » et «Hors norme », transférés du verbal au pictural, suscite l'osmose entre les expressions. «Et s'il y a art... il est dans la sonorité | musicalité... et sa "création franche" | Art sortant des tripes nommé chez les peintres "Hors norme" et qui s'exhibe $\gg .^{29}$ En retour, la peinture est verbalisée par les associations consonantiques de la lettre $\mathrm{R}$ dans «création», «franche», «tripes » et « peintres » faisant écho à la sonorité de « brut ». Le mimétisme entre l'écrit et les formes du Raw Art, autre forme d'art brut, ramène à la source unique de la création. À travers ses appellations, l'art brut domine quantitativement dans le poème. Conférant à l'écrit son iconicité, la nomenclature du catalogue d'art recoupe la fonction organique de l'art brut. Devenue actant principal de la création poétique, « la peinture fait œuvre de poésie », comme dit Louvel. ${ }^{30}$

\section{Apprécier Les dessins de Claudine Goux}

Le Dossier d'Artiste: Claudine Goux composé par Bouraoui inclut un entretien, une série de dessins, des commentaires critiques et le poème intitulé Apprécier les dessins de Claudine Goux. ${ }^{31}$ Goux évoque, dans son entretien avec Bouraoui, l'impact de la peinture et de la théorie de Dubuffet sur son œuvre. Dans le poème, le développement des motifs picturaux de Goux en embranchements rhizomiques est reproduit par l'écrit épousant l'expansion kaléidoscopique des images opérée par le pinceau-stylo au rythme de la musique. « Noir et fin... le trait de Claudine Goux $\mid$ Se fait musicien ». Les notes de musique jouées sur tous les instruments,

\footnotetext{
${ }^{25}$ Hédi Bouraoui, NomadiVivance I. Narratoème, Toronto, CMC Éditions, 2016, p. 11.

${ }^{26}$ Ibid., p. 16.

${ }^{27}$ Ibid., p. 13

${ }^{28}$ Ibid., p. 15.

${ }^{29}$ Ibid., p. 16.

${ }^{30}$ Louvel, L'eil du texte. Texte et image dans la littérature de langue anglaise, Toulouse, Presses Universitaires du Mirail, 1998, p. 69.

${ }^{31}$ Hédi Bouraoui, Dossier d'Artiste : Claudine Goux, in « Revue CMC Review », 6 (2019), 1, p. 13. Les vers cités dans le paragraphe sont tirés du poème.
} 
« libérées [...] éclairent Visages ». Le langage est rendu sonore et le dessin mouvant par les « jeux du trait » à la fois pictural et scriptural animant l'écrit et les images, aboutissant à une expression triplement musicale, littéraire et visuelle : « Chanter poésie | dessins fourmillants ». Le crayon-pinceau du poète, propulsé dynamiquement, inscrit «l'univers sensoriel en ses atouts et des destins », faisant advenir les visions. Telle la figue de Barbarie dans le poème Barbarituifévoqué plus haut, la poussée de l'écrit est végétalisée, processus naturel ne nécessitant pas l'intervention de l'intellect humain.

La densité du sens bourgeonne... et fleurit...

Se mue en oiseaux... et autres couronnes...Alibis

À dévoiler flamboyantes surprises... Hors norme!

À noter que Claudine Goux est unique en...

Ce genre d'art Outsider... de ladite Création Franche

$[\ldots]$

Son expression artistique lui appartient... en Solo.

L’emprunt à la terminologie musicale souligne le génie pluriel de l'artiste, soliste identifiée aux musiciens, dont participe le poète, orchestrant la peinture et la musique dans le poème unifiant les arts.

Selon Louvel, les références aux styles picturaux servent de «pattern » au texte. ${ }^{32}$ L'intégration au poème des appellations Hors norme, Outsider et Création Franche, restituent leurs significations premières aux mots qui les composent. Hors norme s'applique aux «surprises » dévoilées par le pinceau, Outsider souligne sa spécificité par rapport aux autres peintres du mouvement, Création est le modèle de l'écrit poétique imprégné de l'indépendance d'esprit de Goux.

\section{L'interaction du poème et des tableaux: Ma Ville-Reine ${ }^{33}$ et Danse nocturne de Claudine Goux}

Bouraoui signale, à propos de Ma Ville-Reine, que sa poésie se fait descriptive pour représenter son expérience de Toronto où il vit depuis « près d'un demi-siècle ». ${ }^{34}$ On le suit dans sa promenade, dépassant la Tour CN, ${ }^{35}$ le Skydome, la rue Yonge, le métro, North York et Willowdale, jusqu'au bord du lac Ontario. L'itinéraire du

\footnotetext{
${ }^{32}$ Louvel, Le tiers pictural, p. 106.

${ }^{33}$ Bouraoui, NomadiVivance, pp. 19-22.

${ }^{34}$ Ibid., p. 11.

${ }^{35}$ La Tour CN, lieu d'inspiration privilégié, symbole du multiculturalisme canadien, réapparaît à plusieurs reprises dans l'œuvre de Bouraoui.
} 
poète, voyage dans l'espace et le temps, le ramène à l'histoire de la ville et au passé proche de l'« immigré récent » qui redécouvre la ville en se l'appropriant, faisant de Toronto une « Carthage cosmopolite » où il retrouve l'« Écho direct de [son] Ifrikia natale $\gg .^{36}$

Le poème est enchâssé entre deux œuvres picturales de Goux et de Dodik Jégou. La première, Danse Nocturne de Goux (Fig. 1) ${ }^{37}$ sert de frontispice intérieur au recueil. À droite, un musicien jouant du pipeau et du tambour semble éclairé par un projecteur. À gauche, un Amérindien, reconnaissable à sa coiffe de plumes et à sa jupe effrangée, danse au son de la musique. Évoquant les figurines d'un jeu de cartes, les tonalités et les postures sont symétriquement inversées. Les motifs géométriques se redoublent en reflet, les triangles isocèles sur le pourtour du tambour dupliquant les triangles équilatéraux pointés comme des yeux sur la coiffe de l'Indien. Les rayures grises et noires des plumes de la jupe contrastent avec les plis du chapeau du musicien aux deux grandes plumes recourbées reproduisant les doigts du danseur en transe. Le musicien semble absorbé par le maniement de ses instruments. Tenant sa baguette noire et sa flûte blanche à l'horizontale, il rythme la mélodie avec son talon. Les lignes parallèles formées par la baguette et le pipeau, figurent une portée musicale. Sur la surface du tambour, à la fois partition et page d'écriture, les notes blanches alternent avec les notes noires figurées symboliquement par la boule à l'extrémité de la baguette. Les deux personnages représentent ensemble le poète unissant les arts, les cultures et les époques dans sa course effrénée accordée à la frénésie urbaine de «Toronto, Méga-polis la plus croissante d'Amérique $\gg .^{38}$

Comme Goux l'explique à Bouraoui, le mythe d'Orphée a inspiré ses motifs de musiciens. La Tour CN duplique le poète-musicien jouant du chalumeau dans sa marche entre les « myriades de fenêtres illuminées » qui l'entraînent à travers les «labyrinthes des langues » et l'« arc-en-ciel des visages » dans la ville, « lieu de rencontres $\gg$ favorisant les contacts entre les époques, les ethnies et les personnes. ${ }^{39}$ Le poète rencontre d'abord l'Indien drogué, le danseur dessiné par Goux, à qui la parole est donnée : «Un drogué Iroquois maccoste... [...] | -- Aidez-moi... Ils font tout pour toi... Rien pour moi... | l'Iroquois ! $\gg .{ }^{40}$ L'allusion aux autochtones et à leur célèbre mépris du vertige, « au sommet de la Tour » se renforce de la référence à l'histoire canadienne: «Nous avions partagé la chaleur... de la découverte d'Étienne Brulé $\gg .^{41}$ Brulé fut le premier européen qui atteignit la région des

\footnotetext{
${ }^{36}$ Bouraoui, NomadiVivance, p. 19. Le terme Ifrikia ramène au passé lointain du continent africain.

${ }^{37}$ Ibid., p. 17.

${ }^{38}$ Ibid., p. 19.

${ }^{39}$ Ibid., pp. 19-20.

${ }^{40}$ Ibid.

${ }^{41}$ Ibid., p. 20.
} 
Grands Lacs, au $17^{\text {ème }}$ siècle, puis vécut avec les Hurons, devient l'interprète et le guide de Samuel de Champlain et fut tué par une tribu ennemie. Intégré au poème, il figure l'opposition entre la nature et la culture, à partir de laquelle l'art brut a été créé. La discussion entre le poète et l'Amérindien se réfere implicitement aux idées exposées par Dubuffet dans son essai « Honneur aux valeurs sauvages », rendant hommage à «l'art de haute voyance » des Indiens d'Amérique, exemple à suivre pour « l'homme social et policé ». ${ }^{42}$ La même conception se dégage, dans le poème, de la connivence entre le poète-musicien et l'Indien extatique soulevé de terre par le battement lancinant du tambour.

D’autres échanges ponctuent la promenade du poète dans la ville. Structuré par le refrain « Je ne fais que passer », le poème égrène la multiplicité géo-ethnique de Toronto à travers ses rencontres suivantes avec l'« Anglo saoul », dévalorisant le groupe social dominant, la «Franco de Souche», pique ironique aux tergiversations identitaires des Québécois, puis les Zaïrois, en référence à la réalité multiculturelle de la ville. Traité lui-même d'《ethno », appellation dénigrante dans le contexte urbain torontois, le poète incarne les immigrants du monde entier dans la cité ontarienne où les « cent-soixante-neuf langues... d'icitt $\gg{ }^{43}$ recroisent le parler québécois.

La fin du poème est divisée en deux sous-parties, «L'été » et «l'hiver ». Dans la première partie est exalté l'égalitarisme gommant les différences dans la chaleur estivale dont jouit l'ensemble de la population au bord de l'eau. Dans la deuxième partie, les oies sauvages portant dans leur bec « un bouquet de mystère ${ }^{44}$ évoquent le silence dans lequel la colombe au rameau d'olivier, symbole biblique de paix, s'envole pour ne plus revenir. La blancheur estompant les formes des oiseaux, des voiliers et des flocons de neige compose un ensemble monochrome confondant le ciel, la terre et l'eau. Dans le dessin de Dodik Jégoù (Fig. 2), à la fin du poème, la sirène, dernière rencontre du poète, mêle les règnes humain et animal, debout sur sa queue-ventouse, incarnant la Tour $\mathrm{CN}$, fermant la randonnée circulaire du poète dans Toronto.

\section{La traversée transréelle : Le Temple Voilier de Rêva Rémy}

La première de couverture de Traversées est un iconotexte où le titre du recueil, Traversées. Poèmes, est surimposé au tableau de Rêva Rémy Le Temple Voilier (Fig. 3). ${ }^{45}$ Louvel observe à propos des « images qui prennent la place d'un

${ }^{42}$ Dubuffet, L'homme du commun à l'ouvrage, p. 114.

${ }^{43}$ Bouraoui, NomadiVivance, pp. 21-22.

${ }^{44}$ Ibid., p. 22.

${ }^{45}$ Louvel, Le tiers pictural, p. 106. 
titre », qu' elles enclenchent le « passage entre image et texte $\gg \cdot{ }^{46}$ La coprésence du verbal et du visuel sur la première de couverture souligne la continuité entre l'écrit et le motif aquatique récurrent chez Bouraoui. Le titre renvoie au tableau et viceversa, présentifiant le va-et-vient inter médiatique actualisé par l'assimilation de l'écriture à la peinture à l'eau, activée par l'intuition, « bulle d'oxygène linguistique qui fait flotter le poème dans les sphères mystérieuses du suggestif $\gg .{ }^{47}$ Dans le tableau de Rémy, le triangle formé par l'escalier et sa rampe figure une voile et un mât reflétés dans l'eau. Inscrits par et sur l'eau-encre, les poèmes s'apparentent « aux aquarelles où l'eau, glissant sur la feuille [...] fait danser couleurs, ombres et lumières $\gg .^{48}$ L'écriture confondue à la peinture métaphorise la poésie devenue navigation. Les mots sont figurés par les pirogues blanches au fond arrondi, en forme de croissant de lune, balancés sur l'eau verte. La création poétique est un voilier instable, assimilé également tantôt à un train express, tantôt à un vol d'oiseau : « Au-delà du TGV de mes pensées | Je m’envole plume allègre | Ma ligne de mire se fait aquarelle $\gg .{ }^{49}$ Tracée à la plume, l'écriture poétique dessine, en même temps que les mots, le vol imprévisible de l'oiseau. Écrire est hasardeux, s'agissant d'une « traversée » à la destination indéterminée. Le Transréel bouraouïen naît de l'effet conjugué du visuel et du scriptural faisant naître le poème.

Telle encre à investir la page

Je méandre à la recherche de l'insolite

Le Blanc refuse de baisser le caquet

Je louvoie... fragmente... rature

Les Couleurs chantournent une fantaisie

$[\ldots]$

Émerge peu à peu l'équilibre de l'aube

$[\ldots]$

L'aquarelle se miracle objet d'art ${ }^{50}$

Sur le modèle de l'écoulement spontané d'eau colorée sur la toile, l'écriture est rendue fluide par les points de suspension marquant les stades de l'inscription sur la page contrée par le «Blanc » et les « Couleurs » dotés de volonté autonome. Contournant tous les obstacles qu'ils lui opposent de front, le poète-navigateur, usant du néologisme, « je méandre », se laisse porter par le fleuve peint par Rémy jusqu’à ce que « la dérive du temps récupère la perle » de « l'instantané ».51

${ }^{46}$ Ibid.

${ }^{47}$ Bouraoui, En amont de l'intuition, p. 6.

${ }^{48}$ Bouraoui, Traversées, p. 5.

${ }^{49}$ Ibid., pp. 12-13.

${ }^{50}$ Ibid., pp. 14-15.

${ }^{51}$ Ibid., pp. 12-13. 
La métaphorisation de l'écriture poétique en peinture à l'eau reconstitue le processus créateur décrit par Dubuffet comme «les combats intervenus entre l'artiste et les indocilités des matériaux qu'il a mis en œuvre $\gg .{ }^{52}$ La peinture est « une aventure dont on ne sait où elle vous conduira », dit Dubuffet, décrivant l'artiste « attelé avec le hasard $\gg .{ }^{53}$ La vitalité de l'œuvre d'art est relative à la liberté du matériau insoucieux des «intentions concertées de l'artiste $» .^{54}$ Le peintre, maniant «des matières magiques,$^{55}$ doit « respecter les impulsions, les spontanéités ancestrales de la main humaines quand elle trace ses signes », livrant «crues [...] humeurs et impressions $\gg .{ }^{56}$ Conformément au mot d'ordre de Dubuffet, « Que la raison bascule », ${ }^{57}$ le poète « rame dans l'opaque », telle une barque livrée aux remous marins : « Les courants te saluent $|[. .]$.$| Et dans l'estuaire$ du poème $\mid \mathrm{Tu}$ te déverses $\gg .{ }^{58} \mathrm{La}$ métaphore filée retrace la navigation hasardeuse de l'écriture-aquarelle sur la page dans le sillage de l'intuition.

\section{Adam Nidzgorski et la nomadanse}

L'écriture équivaut à la nomadanse, néologisme désignant la « danse artistique, esthétique de vivre le pluriel cadencé de l'Être et de l'Avoir, des éléments de la Terre et $\mathrm{du}$ Ciel, de toutes les contingences dans l'univers de l'actuel $\gg .{ }^{59}$ Abderrahman Beggar note à ce propos que « le nomadanseur accumule au lieu de limiter pour contenir $\gg .{ }^{60}$ Le danseur stylisé sur le dessin d'Adam Nidzgorski (Fig. 4), à la page liminaire de la seconde section de Traversées, Transit en Art Majeur, représente le poète dans sa nomadanse, rassemblant la diversité des sensations et des expériences dans une écriture totalisante. De navigation, la poésie est une chorégraphie marine dans «Art écume » où l'impétuosité des vagues reproduit l'élan de l'intuition en référence à l'art brut : « Brute la vérité de l'art | Fait semblant de cacher | Ondes de choc et liberté de mains », l'écriture résultant de la projection d'encre-eau traduisant la tempête intérieure du poète, lorsque

\footnotetext{
${ }^{52}$ Dubuffet, L'homme du commun à l'ouvrage, p. 26.

${ }^{53}$ Ibid., pp. 26-27.

${ }^{54}$ Ibid., p. 31.

${ }^{55}$ Ibid., p. 34.

${ }^{56}$ Ibid., p. 44.

${ }^{57}$ Ibid., p. 53.

${ }^{58}$ Bouraoui, Traversées, p. 32.

${ }^{59}$ Bouraoui, NomadiVivance, p. 13

${ }^{60}$ Abderradam Beggar note à ce sujet que « L’Un représenté par la norme est maintenant soumis au divers. Le nomadanseur accumule au lieu de limiter pour contenir », Abderrahman Beggar, La Transpoétique et le normatif chez Hédi Bouraoui, dans Continents manuscrits 2 (2014), pp. 1-10, p. 6.
} 
«Dans le déferlement de l'écume | Se dénouent des fondations arc-en-ciel ». ${ }^{61}$ Comme dans la peinture intuitiste, appuyée sur la ligne courbe, ${ }^{62}$ le poème se décante en reproduisant les formes contournées de l'écume aux « volutes en nœuds papillons $\gg,{ }^{63}$ ainsi, dans le mouvement du crayon inscrivant le poème-dessin régit le mouvement général des courbes, des pleins et des déliés de l'écriture animée. La succession cadencée des mots, « éclats d'étreintes multicolores », et des blancs, « souffles prodigieux du silence » ${ }^{64}$ vivifie les signes d'écriture. Leur mouvance est reproduite dans le dessin de Nidzgorski où les lignes dansent, ondulant comme les vagues d'où émerge la tête du poète-nageur qui se «laisse aller», au gré de «l'instantané de $[\mathrm{sa}]$ vision $\gg .{ }^{65}$

Circulent paroles en amont

Du Rituel qui guérit

Recharge art et vie du fortuit !

$[\ldots]$

Ni genre ni barrière ni mise en scène

Les mots vécus se font chair intérieure

Nous sommes dans la Vérité de nous-mêmes. ${ }^{66}$

La spontanéité de la danse ramène à la conception de Dubuffet du hasard l'emportant sur le calcul raisonné pour l'artiste soumis au caprice de son matériau doté de volonté propre. Manifester l'intuition, c'est, selon Dubuffet, « respecter les impulsions, les spontanéités ancestrales de la main humaine $\gg,{ }^{67}$ afin qu' « humeurs et impressions [soient] livrées crues $\gg .{ }^{68}$ La poésie-nomadanse réfracte les expressions verbale et visuelle de la spontanéité :

Un dialogue intérieur se développe

Devient chant polyphonique au rythme

Du pinceau aller-retour dans l'air du temps. ${ }^{69}$

La polyphonie, désignant chez Dubuffet le « chœur de toutes les composantes de la pensée de l'artiste $\gg,{ }^{70}$ est pour Bouraoui l'expression totalisante du dialogue

${ }^{61}$ Bouraoui, NomadiVivance, p. 43.

${ }^{62}$ Sivry, Pour un art de l'intuition, p. 223.

${ }^{63}$ Bouraoui, NomadiVivance, p. 43.

${ }^{64}$ Ibid.

${ }^{65}$ Ibid., p. 48.

${ }^{66}$ Ibid., pp. 44-45.

${ }^{67}$ Dubuffet, L'homme du commun à l'ouvrage, p. 34.

${ }^{68}$ Ibid., p. 44.

${ }^{69}$ Bouraoui, NomadiVivance, p. 45.

${ }^{70}$ Dubuffet, L'homme du commun à l'ouvrage, p. 28. 
entre la peinture, la poésie, la danse et la musique : « Et qui fait la performance | Sinon la nomadanse...des mots! $\gg$, s'interroge-t-il dans Passerelles. ${ }^{71}$ La nomadanse de l'écrit et des images actives fait fusionner les expressions artistiques, «En Nomadanses de Formes... de Figures $\gg,{ }^{72}$ comme dans la danse des « Mots aimantés de musique | Dansée dans l'arrière-boutique | De l'Univers » que «L'Homme s'amuse à $[\ldots]$ accorder $» .^{73}$

\section{La poésie, « art de la surprise » $(\text { Sivry })^{74}$}

Notre étude visait à souligner la similarité entre les conceptions respectives de Bouraoui et de Dubuffet, et, plus spécifiquement, leur même approche de la réalité, « intuitive-expérimentale ». ${ }^{75}$ Comme dans le tableau de Goux où, côte-à-côte, le poète-musicien et le danseur Iroquois font doublement référence à l'art Native American qui a inspiré Dubuffet et au transculturalisme de Bouraoui, écrivain torontois, ainsi une poḯtique de la création se dégage de la coprésence de l'écrit poétique et des tableaux rattachés à l'art brut. L'intuition, source, selon Bouraoui, de « traversées insoupçonnées » et de « dialogues incessants $\gg,{ }^{76}$ s'origine dans les «voix intérieures $\gg^{77}$ mises en œuvre dans l'art selon Dubuffet. Le poète, «Ballotté entre Intuition et Imagination ${ }^{78}$ conçoit la poésie comme « un dialogue intérieur » à l'opposé de «la Beauté monnayée », ${ }^{79}$ boiteuse et sans avenir. La poésie, «langue du monde » pour Sivry, ${ }^{80}$ synthèse des sensations intérieures et extérieures, émane, dit Bouraoui, de « L'intuitif... seul à égaliser | Les plus sensibles... les plus prévoyants... $\gg .^{81}$

Dans son commentaire sur l'exposition Monet et l'abstraction qui a eu lieu à Paris en 2010, Sivry observe qu' «à un moment donné », ce n'est plus l'objet qui est représenté comme dans la peinture figurative, mais « l'acte de peindre luimême $\gg .{ }^{82}$ Ainsi, dit-il, en se référant aux tableaux et aux sculptures intuitistes qui

\footnotetext{
${ }^{71}$ Hédi, Bouraoui, Passerelles. Poésie, Toronto, CMC Éditions, 2018, p. 8.

${ }^{72}$ Ibid., p. 7.

${ }^{73}$ Bouraoui, Traversées, p. 47.

${ }^{74}$ Sivry, Pour un art de l'intuition, p. 38.

${ }^{75}$ Jean H. Duffy, Dubuffet Plays Hide-and-Seek : Lineage, Reflexivity, and Perception in "Coucou Bazar", in «The Art Bulletin », 98 (2016), 2, pp. 237-260, p. 2.

${ }^{76}$ Bouraoui, Traversées, p. 4.

${ }^{77}$ Dubuffet, L'homme du commun à l'ouvrage, p. 115.

${ }^{78}$ Bouraoui, Passerelles, p. 11.

${ }^{79}$ Bouraoui, Traversées, p. 45.

${ }^{80}$ Sivry, Pour un art de l'intuition, p. 41.

${ }^{81}$ Bouraoui, En amont de l'intuition, p. 34.

${ }^{82}$ Sivry, Pour un art de l'intuition, p. 165.
} 
«manifestent des intuitions créatrices fugaces $\gg,{ }^{83} \mathrm{en}$ va-t-il du peintre intuitiste, appliqué à « laisser l'oeuvre se créer d'elle-même, à partir [du] contact sensoriel et émotionnel instantané et direct avec le monde et la matière $\gg .{ }^{84}$ Faisant doublement écho à la conception de Sivry et à la description de l'artiste par Dubuffet, imprimant « les traces les plus immédiates qu'il se peut de sa pensée et des rythmes et impulsions qui battent ses artères $\gg,{ }^{85}$ la poésie de Bouraoui, incorporant l'art brut et privilégiant les sensations et la surprise, donne à voir la création en acte, exaltant les instincts et les passions qui la vivifient.

\section{OEuvres citées}

Abderrahman Beggar, La Transpoétique et le normatif chez Hédi Bouraoui, in «Continents manuscrits », 2 (2014), pp. 1-10.

Hédi Bouraoui, En amont de l'intuition. Poèmes, Toronto, CMC Éditions, 2013.

Hédi Bouraoui, Traversées. Poèmes, Toronto, CMC Éditions, 2010.

Hédi Bouraoui, L'intuition à l'auvre, in Id. En amont de l'intuition. Poèmes, Toronto, CMC Éditions, 2013, pp. 6-8.

Hédi Bouraoui, Inside Faces-Visages du dedans. Visiotexts/Visionèmes Paintings by/Peintures de Micheline Montgomery, Toronto, CMC Éditions, 2014.

Hédi Bouraoui, NomadiVivance I. Narratoème, Toronto, CMC Éditions, 2016.

Hédi Bouraoui, Passerelles. Poésie, Toronto, CMC Éditions, 2018.

Hédi Bouraoui, Dossier d'Artiste: Claudine Goux, in « Revue CMC Review », 6 (2019), 1.

Isabelle Danto, Quand l'art brut redessine l'art contemporain, in « Esprit », (MarsAvril 2015).

Jean Dubuffet, L'homme du commun à l'ouvrage, Paris, Gallimard, 1991 [1973].

Jean H. Duffy, Dubuffet Plays Hide-and-Seek : Lineage, Reflexivity, and Perception in "Coucou Bazar", in «The Art Bulletin », 98 (2016), 2, pp. 237-260.

Liliane Louvel, L'oil du texte. Texte et image dans la littérature de langue anglaise,

${ }^{83}$ Ibid., p. 168

${ }^{84}$ Ibid., p. 167.

${ }^{85}$ Dubuffet, L'homme du commun à l'ouvrage, p. 43. 
Toulouse, Presses Universitaires du Mirail, 1998.

Liliane Louvel, Le tiers pictural, Rennes, Presses Universitaires de Rennes, 2010.

Liliane Louvel, A reading event The Pictorial Third, in Sillages critiques, 21 (2016).

Michael Nerlich, Qu'est-ce qu'un iconotexte? Réflexions sur le rapport texte-image photographique dans La Femme se découvre d'Evelyne Sinnassamy, dans Iconotextes, sous la direction de Alain Montandon, Paris, Ophrys, 1990, pp. 255-302.

Elizabeth Sabiston, Upstream : Hédi Bouraoui's En amont de l'intuition, in « Revue CMC Review », 1 (2014), 1, pp. 1-9.

Mario Selvaggio, Hédi Bouraoui, Passerelles. Poésie, Toronto, CMC Éditions, 2018 [Compte rendu], in «Revue européenne de recherches sur la poésie », 4 (2018), pp. 260-262.

Éric Sivry, Pour un art de l'intuition. Manifeste de l'intuitisme, Paris, Hermann Éditeurs, 2016.

Peter Wagner, Icons - Texts - Iconotexts. Essays on Ekphrasis and Intermediality, Berlin-New York, Walter de Gruyter, 1996.

\section{Sitographie}

Marilyne Bertoncini, Poésie-première 72 : l'intuitisme [Compte rendu], in Recours au Poème.

$<$ https ://www.recoursaupoeme.fr/poesie-premiere-70-lintuitisme/> 


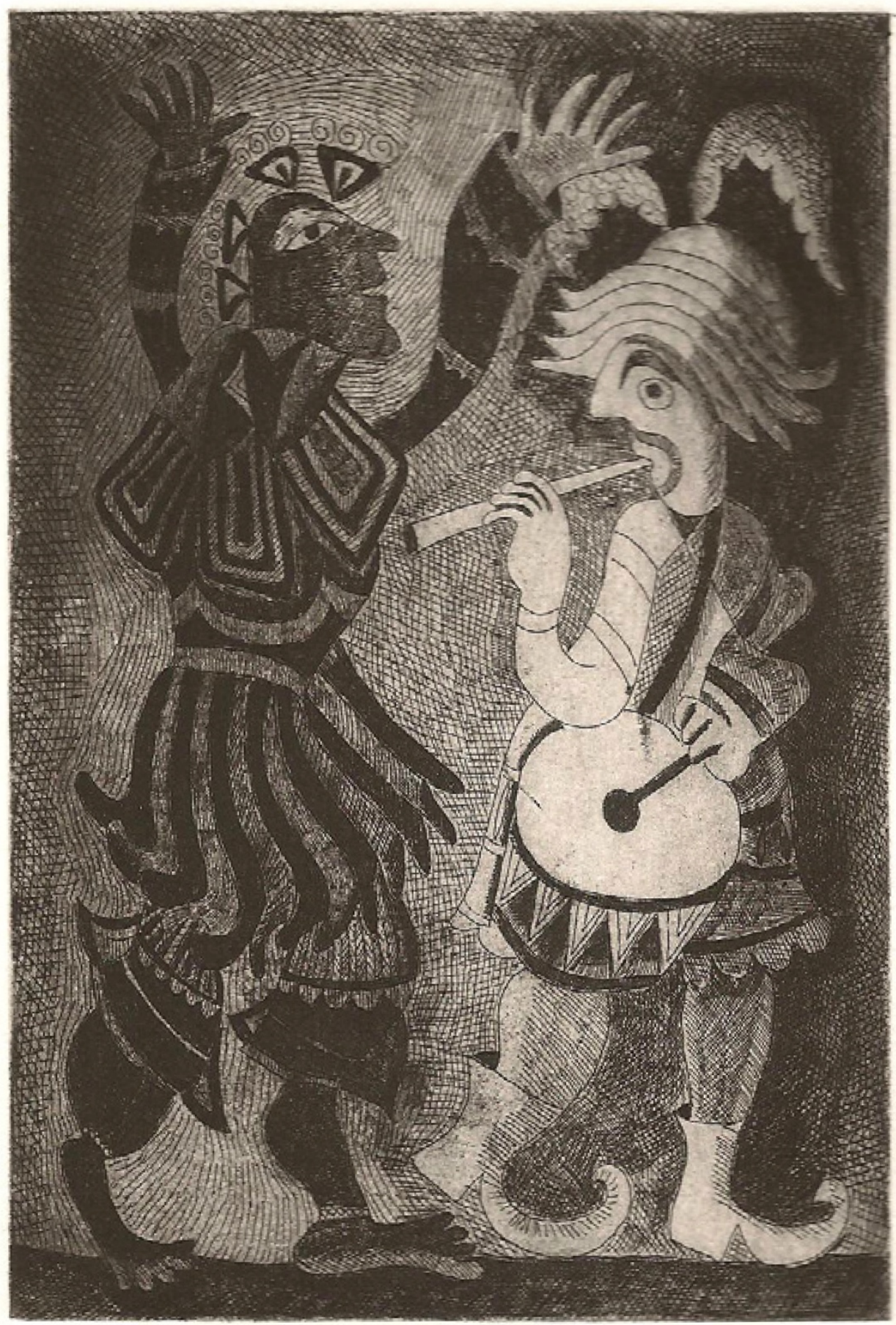

Figure 1 : Claudine Goux, Danse Nocturne, dans Hédi Bouraoui, NomadiVivance I. Narratoème, Toronto, CMCÉditions, 2016, [p. 17]. 


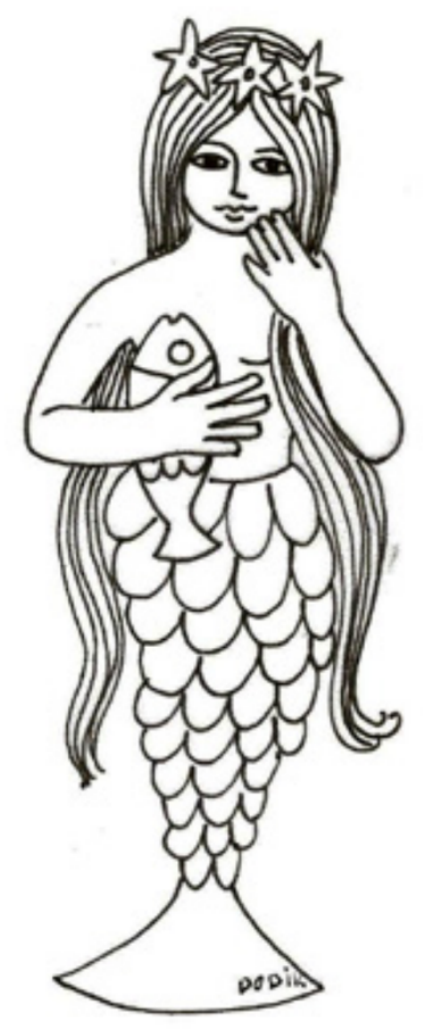

Dodik Jégou

Figure 2 : Dessin de Dodik Jégou dans dans Hédi Bouraoui, NomadiVivance I. Narratoème, Toronto, CMCÉditions, 2016, p. 22. 


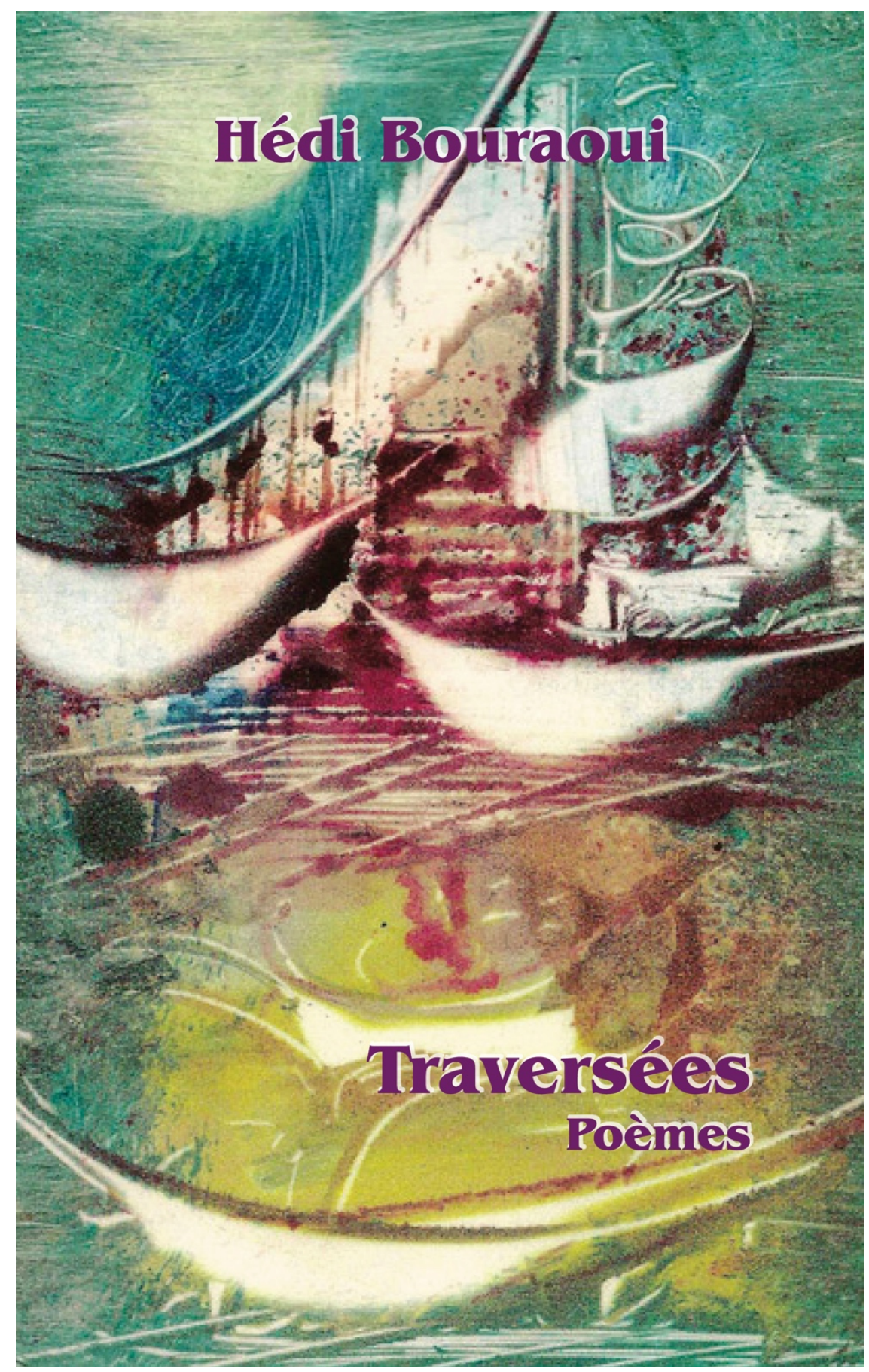

Figure 3 : Première de couverture de Hédi Bouraoui, Traversées. Poèmes, Toronto, CMC Éditions, 2010, composé du titre surimposé au tableau de Rêva Rémy Le Temple Voilier. 


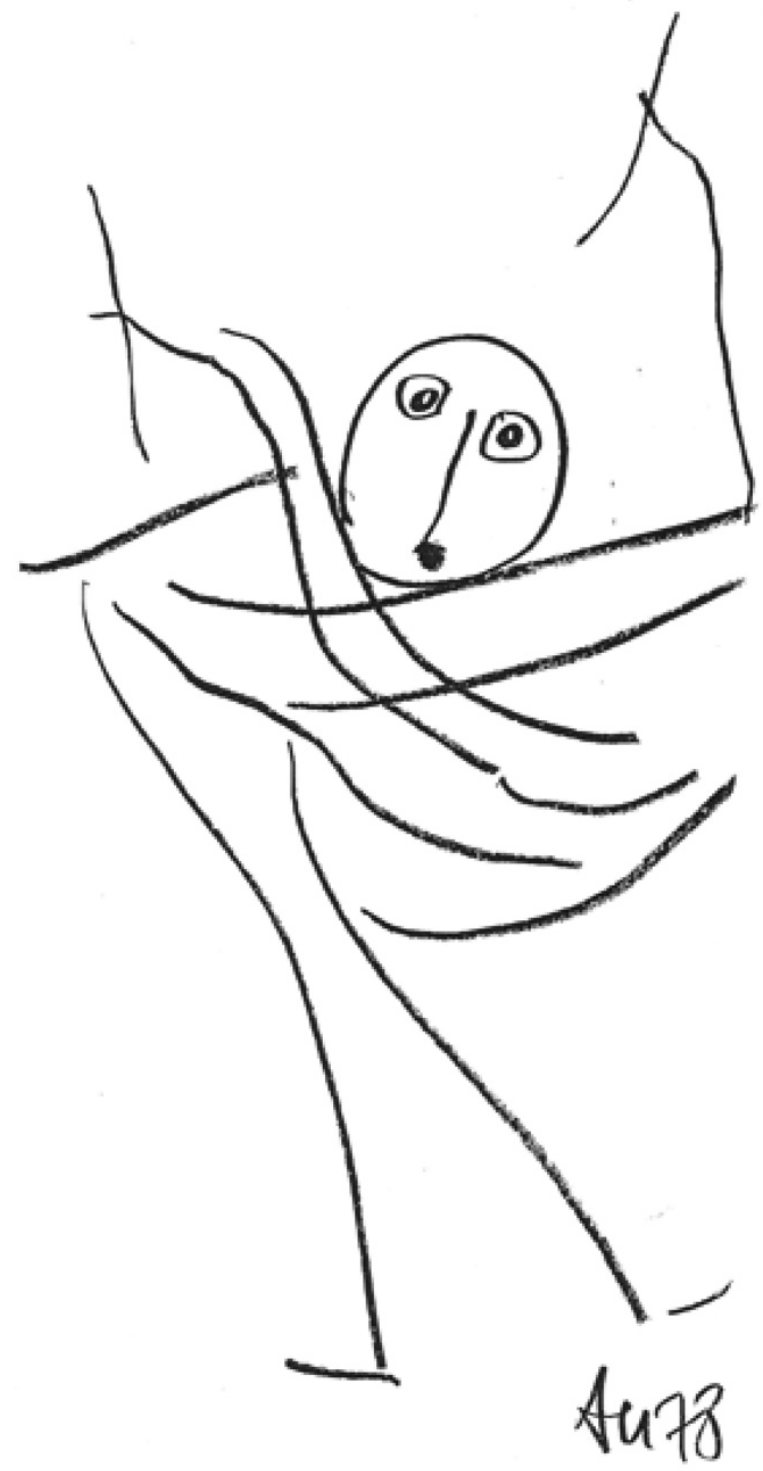

Figure 4 : Dessin d'Adam Nidzgorski dans Hédi Bouraoui, Traversées. Poèmes, Toronto, CMC Éditions, 2010, [p. 41]. 